\title{
A New Optimization Difference Algorithm Based on Fly Fruit
}

\author{
Min Zhang \\ School of Resources and Geosciences. \\ China University of Mining \& Technology , \\ Xuzhou 221116, China
}

\author{
Shouhua Dong \\ China University of Mining \& Technology , \\ Xuzhou 221116, China
}

\begin{abstract}
Acoustic wave equation is an important basis of seismic wave propagation,imaging and migration. Finite difference methods develop with its numerical stability and grid dispersion problems, and the issue of dispersion is a vital above all. Optimization methods can provide a high accuracy within a large wavenumber or frequencies. To obtain a higher precision, this paper propose a optimization difference algorithm based on fly fruit on a wide wavenumber range and construct the function of fitness which makes a process of iterating and finding the best smells. Dispersion analysis demonstrate that this scheme is much better than Taylor-expansion method. We make a experiment by applying homogeneous model, and the results indicate that fly fruit optimization approach a higher accuracy and reduce the computation cost.
\end{abstract}

Keywords: fly fruit algorithm; difference coefficient; accuracy; dispersion

\section{INTRODUCTION}

Seismic numerical simulation, an important fundamental in explorations by the basis of knowing or assuming of the underground media and physical parameters, can be applied to modeling and forecasting the seismic response characteristics in the underground. There are many different fields which have been widely utilized, such as mineral resources, engineering, even environment. Meanwhile seismic numerical simulation can help us get a correct understanding of different geological characteristics on seismic records, which brings out a comprehensive recognition of seismic wave propagation characteristics in various media, eventually forms an effective means of explanation and inversion of the real underground media structure. It also plays a key role in guiding in processing and interpretation of seismic data. Finite difference(FD) method is one of the main numerical methods for solving the problems of given values in the initial and boundary, which has long been applied for its high computational efficiency, low memory cost and simple realization in different difference formats and flows ${ }^{[2]}$.

FD is mainly based on the procedure of difference equations approximating differential equations, namely by substituting the each discrete point value of space, and then the complex differential problems can be solved by algebra problems. However, FD methods develop with its numerical stability and grid dispersion problems, and the issue of dispersion is a vital above all. Numerical dispersion refers to the frequency components of different wavelengths propagate at different speeds, and the actual performance in seismic wave is that the numerical phase velocity differs from its real subsurface phase velocity. Moreover it leads to a low precision in forward modeling for subsequent data processing, interpretation and inversion. Thus the matter of suppressing seismic wave numerical dispersion has become a key issue ${ }^{[3]}$.

Many studies are based on the inhibition of both time and space to achieve high-precision positive dispersion modeling due to the fact that seismic wave spreads in the time and space. Recent studies ${ }^{[4]}$ propose the trust region to determine the optimal implicit FD operators in time-space domain, and the result shows that it reaches a bigger time step in the same modeling accuracy. Liu and Sen propose a new scheme for one-dimensional scalar wave equation based on the timespace domain dispersion relation and plane wave theory, which is made to satisfy the exact dispersion relation ${ }^{[5]}$. According to the algorithm of least square optimization, a new method is developed ${ }^{[6]}$ with excellent suppressing dispersion result. What is more, the regularizing technique to tackle the ill-conditioned property of the symmetric linear system and to stably provide approximate solutions of the FD coefficients for acoustic-wave equations ${ }^{[7]}$, also satisfies the dispersion relationship over a much wider range of frequencies and angles of propagation and can ensure FD coefficients being solved via a well-posed linear system and hence improve the forward modeling precision. In this paper, we propose the fly fruit optimization algorithm(FOA)to obtain globally optimal FD coefficients over the maximum bandwidth for second-order spatial derivatives ${ }^{[8]}$. Further, the results indicate that FOA can approach high precision and less dispersion.

\section{METHOD THEORY}

We start with 2 dimension(2D) acoustic wave equation:

$$
\frac{\partial^{2} \mathrm{u}}{\partial \mathrm{x}^{2}}+\frac{\partial^{2} \mathrm{u}}{\partial \mathrm{y}^{2}}=\frac{1}{v^{2}} \frac{\partial^{2} \mathrm{u}}{\partial \mathrm{t}^{2}}
$$

Where $\mathrm{u}$ is the wave field, $\mathrm{v}$ is the $\mathrm{p}$ wave velocity. Then we propose an FD operator involved $2 \mathrm{M}+1$ points is expressed as

$$
\frac{\partial^{2} \mathrm{u}}{\partial \mathrm{x}^{2}} \approx \frac{1}{\mathrm{~h}^{2}}\left[\mathrm{c}_{0} \mathrm{u}(\mathrm{x})+\sum_{\mathrm{m}=1}^{\mathrm{M}} \mathrm{c}_{\mathrm{m}}(\mathrm{u}(\mathrm{x}+\mathrm{mh})+\mathrm{u}(\mathrm{x}-\mathrm{mh}))\right]
$$

Where $\mathrm{x}$ is a certain real variable, $\mathrm{h}$ is the spatial step, ${ }^{c_{m}}$ are FD coefficients. 
First, according to the Liu's derivation by plane wave theory $^{[5]}$, we let

$$
\mathrm{u}(\mathrm{x}+\mathrm{mh})=\mathrm{u}_{0} \mathrm{e}^{\mathrm{ik}(\mathrm{x}+\mathrm{mh})}
$$

Where $\mathrm{u}_{0}$ is a certain constant value, $\mathrm{i}$ is the imaginary unit and $\mathrm{k}$ is the wavenumber. Substitute Eq. (3)into (2) and it easily obtains

$$
\mathrm{k}^{2} \approx \frac{1}{\mathrm{~h}^{2}}\left[\mathrm{c}_{0}+2 \sum_{\mathrm{m}=1}^{\mathrm{M}} \mathrm{c}_{\mathrm{m}} \cos (\mathrm{mkh})\right]
$$

Besides,

$$
\lim _{\mathrm{k} \rightarrow 0}\left[\mathrm{k}^{2} \mathrm{~h}^{2}+\mathrm{c}_{0}+2 \sum_{\mathrm{m}=1}^{\mathrm{M}} \mathrm{c}_{\mathrm{m}} \cos (\mathrm{mkh})\right]=\mathrm{c}_{0}+2 \sum_{\mathrm{m}=1}^{\mathrm{M}} \mathrm{c}_{\mathrm{m}}=0
$$

Then let

$$
\gamma=\mathrm{kh}, \cos (\mathrm{mkh})=\cos (m \gamma)
$$

Substitute Eq. (5)and (6)into(4) and it obtains

$$
\left[2 \sum_{\mathrm{m}=1}^{\mathrm{M}} \mathrm{c}_{\mathrm{m}}(1-\cos (\mathrm{m} \gamma))\right]-\gamma^{2} \approx 0
$$

Where $\gamma$ ranges from 0 to $\pi$.

Meanwhile within the range of a certain wavenumber $k_{0}$, we use square error

$$
\text { Error }=\int_{0}^{\mathrm{k}_{\mathrm{o}}}\left\{\left[2 \sum_{\mathrm{m}=1}^{\mathrm{M}} \mathrm{c}_{\mathrm{m}}(1-\cos (\mathrm{m} \gamma))\right]-\gamma^{2}\right\}^{2} \mathrm{~d} \gamma
$$

Liu and Sen ${ }^{[5]}$ get second-order spatial FD coefficients $c_{m}$ by adopting the Taylor series explanation minimization(TEM )

$$
c_{m}=\frac{(-1)^{m+1}}{m^{2}} \prod_{1 \leq n \leq M, n \neq M}\left|\frac{n^{2}}{n^{2}-m^{2}}\right|, \quad m=1,2,3 \ldots, M
$$

We propose the FOA method to solve Eq. (8)

Let fitness function

$$
\text { Smelli }=\min \left\{\int_{0}^{\mathrm{k}_{\mathrm{o}}}\left\{\left[2 \sum_{\mathrm{m}=1}^{\mathrm{M}} \mathrm{c}_{\mathrm{m}}(1-\cos (\mathrm{m} \gamma))\right]-\gamma^{2}\right\}^{2} \mathrm{~d} \gamma\right\} \text {, (10) }
$$

Subject to $\gamma \in(0, \pi)$

Where Smelli is the object function, $c_{m}$ come from the random initial fruit fly swarm location and its smell of food in random directions and distances.After iterating and finding the best smell, the FD coefficients can be obtained.

\section{ACCURACY ANALYSIS}

We utilize the absolute error(Eq. (8)) to compare the TEM with FOA algorithm. Fig.1 shows variations of absolute error of the space-domain dispersion relation with $\gamma$ and its magnification by the TEM and the FOA, respectively. $M=4$ and $\mathrm{k} 0=1.16$ are given. Further when $\mathrm{M}=8$ and $\mathrm{k} 0=2.10$, Fig. 2 also shows variations of absolute error of the space-domain dispersion relation with $\gamma$ and its magnification by the TEM and the FOA, respectively. Seeing from Fig. 1, we can get the results that

i The longer the length of difference operator, the higher of the accuracy.
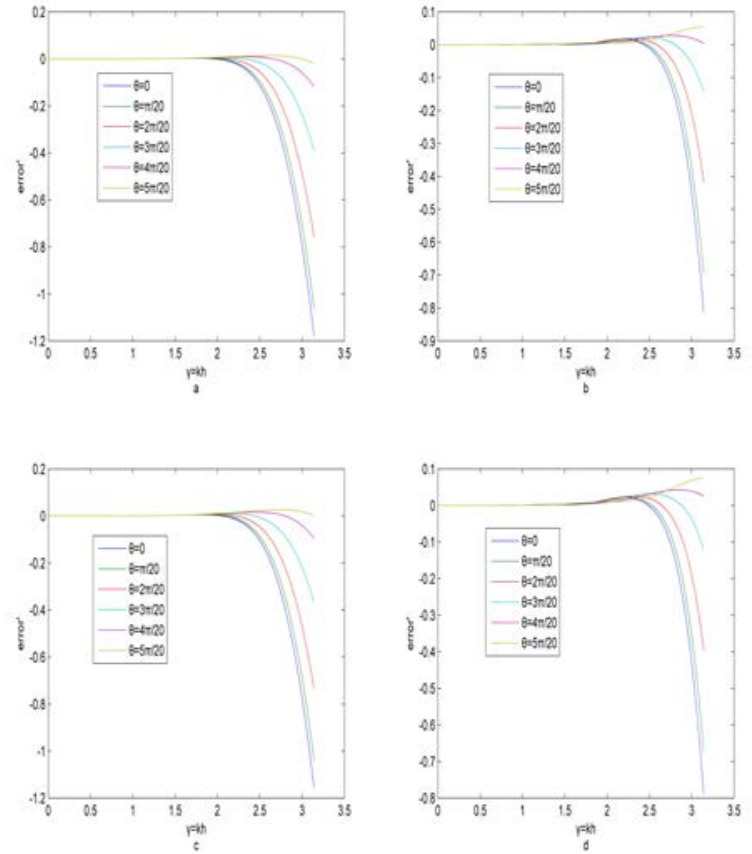

Fig.1. the absolute error difference between TEM and FOA(M=4,the right side is the magnification)

ii For the same wavenumber range, the FOA has a small error than the TEM.

\section{DISPERSION ANALYSIS}

We apply the following formula ${ }^{[5]}$ to determine timespace-domain dispersion relation based on spatial FD coefficients for 2D acoustic FD modeling,

$$
\begin{aligned}
& \sum_{m=1}^{M} c_{m}(2-\cos (m k h \cos \theta)-\cos (m k h \sin \theta))- \\
& r^{-2}(1-\cos (r k h)) \approx 0,
\end{aligned}
$$

Where $r$ equals to $v \tau / h, \tau$ is the time step, and ${ }^{\theta}$ is the propagation direction angle of the plane wave.

\section{NUMERICAL EXAMPLE}

We give an examples of 2D acoustic FD modeling for a homogeneous model, where $v=2000 \mathrm{~m} / \mathrm{s}, \mathrm{T}=0.0005 \mathrm{~s}$, and the operator length of FOA is 8 , compared with TEM, M equals to 8,15 , and 16 , respectively. And the size of calculation is $250 \times 250$, also the spatial grid is $10 \mathrm{~m}$. Ricker wavelet with main frequency of $20 \mathrm{~Hz}$, located in the center of the model, is used to generate vibrations, the vibration function is

$$
s(t)=\left(1-2\left(\pi^{2} \times f_{0}^{2} \times\left(t-t_{0}\right)^{2}\right)\right) \times e^{\left(-\left(\pi^{2} \times f_{0}{ }^{2} \times\left(t-t_{0}\right)^{2}\right)\right)}
$$

where $\mathrm{t} 0=40 * \mathrm{~T}$.

Fig.2 shows the snapshots at $\mathrm{t}=350 \mathrm{~ms}$ and their differences of acoustic FD modeling for a homogenerous model using second-order temporal FD and (2M)th-order spatial FD from the TEM and the FOA of minimizing the absolute error of 2D time-space-domain dispersion relation, respectively. Moreover, it reveals that the operator length can 
be decreased significantly when using the FOA, compared to the TEM, for the same modeling precision. From Fig.2, it can be indicated that:

The modeling accuracy of the FOA with $\mathrm{M}=8$ (Fig.2d) is much greater than the TEM with $\mathrm{M}=8$ (Fig.2a), and is nearly the same as the TEM with $\mathrm{M}=16$ (Fig.2c).

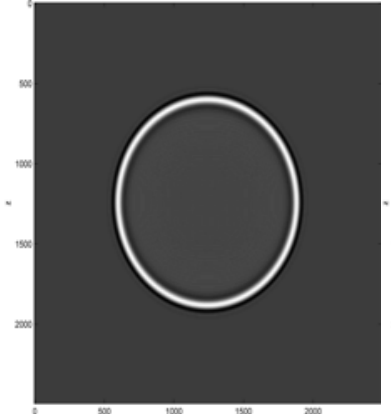

a

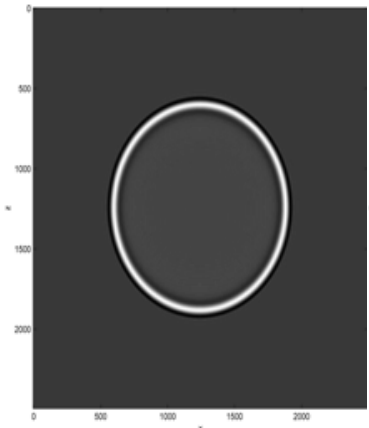

C

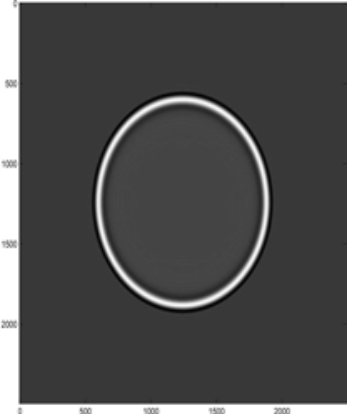

$\mathrm{b}$

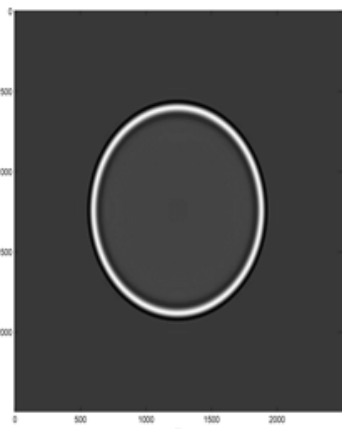

d
Fig.2. Snapshots at $\mathrm{t}=350 \mathrm{~ms}$ and their differences of acoustic FD modeling for a homogeneous model from TEM and FOA. (a, b, c) Are by the TEM, $\mathrm{M}=8,15,16$,respectively. (d) Is by the FOA, $M=8$.

\section{CONCLUSIONS}

This paper, we have developed a new FOA-based scheme to obtain FD coefficients over the maximum bandwidth for second-order spatial derivatives based on minimizing the absolute error of space-domain dispersion relation. It is easily to calculate the optimal FD coefficients when the FOA is applied. Compared with the conventional published TEMbased coefficients, FOA-based coefficients provide a great less dispersion error over the same wavenumber range with the same spatial FD operator length. Accuracy analyses and modeling examples prove the excellent advantages of the proposed method.

\section{ACKNOWLEDGEMENTS}

We thank Prof. Wen-Tsao Pan for providing their excellent FOA MATLAB codes to implement this research.

\section{VIII.REFERENCES}

[1] Virieux, J.SH-wave propagation in heterogeneous media: Velocity stress finite-difference method[J].Geophysics, 1984,49:1933-1957.

[2] Virieux, J. P-SV wave propagation in heterogeneous media: Velocity stress finite difference method[J]. Geophysics, 1986,51: 889-901.

[3] Yang, D. H., G. J. Song, B. L. Hua, and H. Calandra, Simulation of acoustic wavefields in heterogeneous media: A robust method for automatic suppression of numerical dispersion: Geophysics, 2010,75, no. 3, T99-T110

[4] Liang W Q, Wang Y F, Yang C C. Determination on the implicit finitedifference operator based on optimization method in time-space domain[J].Geophysical Prospecting for Petroleum,2015,54(3):254-259.

[5] Liu, Y., and M. K. Sen, 2009c, A new time-space domain high order finite-difference method for the acoustic wave equation: Journal of Computational Physics, 228, 8779-8806.

[6] Liu, Y., 2013, Globally optimal finite-difference schemes based on least squares: Geophysics, 78, no. 4, T113-T132.

[7] Y F Wang, W Q Liang, Z Nashed, X Li, G H Liangand C C Yang, Seismic modeling by optimizing regularized staggered-grid finitedifference operators using a time-space domain dispersion relationship preserving method, Geophysics, Vol. 79, No.5, T277-T285.

[8] W.-T. Pan, A new fruit fly optimization algorithm: Taking the financial distress model as an example, Knowl. Based Syst. 26 (2012) 69-74. 